\title{
Computing Platform \& Application Service Integrated By Green And Efficiency Method Is The Key Of Successful Cloud Service
}

\author{
Yue Zhou \\ National Application Testing Labs \\ Beijing, China \\ e-mail: zhouyue@bsw.net.cn
}

\author{
Wenchuang Qin, Nafei Zhu \\ National Application Testing Labs \\ Beijing, China \\ e-mail: qinwenchuang@bsw.net.cn \\ zhunf@bsw.net.cn
}

\begin{abstract}
In the vigorous developing period of the cloud application, it is necessary to identify the application services, cloud computing platform and the relationship with the energy efficiency. Application service is the foundation, the cloud architecture is the platform, and the energy efficiency of the combination of them is the key to the success of the cloud services. The cloud computing architecture is not necessarily to solve the problem of application performance, and expanding the application of cloud computing platform service in the manner of inefficient and out-of-order can only cause the huge waste of social resources, including electric power, network and repeated investment. The viewpoint of this article is that cloud application should be based on application service, and only the green and efficient integration of the cloud platform can build a good ecological environment for the cloud application
\end{abstract}

Keywords-component; application service; cloud computing, application performance; electric power resource; network resources

\section{INTRODUCTION}

Cloud computing is recognized to be another major information technology innovation after the personal computer and the Internet. China's national economic and social development plan make the cloud computing which is the representative of a new generation for information technology be key development for the strategic emerging industry. With cloud computing mode for service characteristics in all applications will get rapid development [1].

First, three operators of telecom industry will speed up the implementation of cloud computing strategy to achieve business innovation with the aid of cloud computing.

Second, in the financial industry small and medium-sized financial industry force set up public cloud service center.

Third, government promotes the reform of electronic government through the cloud computing platform.

Fourth, education industry improves resource utilization by establishing sharing cloud platform.

While cloud computing develops vigorously, we should keep calm to avoid waste investment and repeated construction and so on.

\section{The APPlicAtion Of Cloud COMPUTING NeEDS CONSIDERATION}

The concept of cloud computing proposed to satisfy the needs of integration of computing resources and services is now the strategic high ground in the international competition of information technology. In the past two years, more and more people are talking about cloud computing and various institutions invest a lot of material and financial resources to cloud computing. At the same time, a variety of cloud computing and data center base are built. To promote the healthy development of cloud computing and standardize the cloud computing industry development, it needs to seize the development opportunities and also calmly analysis some problems in reality.

The following problems for the development of cloud computing are worth our careful thinking of.

\section{A. Cloud Computing Cannot Resolve All The Performance Problems Of Application Services}

Laying much more attention on hardware than that on the software has been the serious problem of China's information construction. This because buying hardware is easy, but incorporate hardware into their business systems and IT architectures to construct a high performance IT application service platform is not easy. Many enterprises in the face of system performance bottlenecks often think to improve the hardware performance firstly, which is to improve performance through a stack of hardware, and even think that if they bought a lot of cloud computing service resources they can solve the application performance problems. But the fact is often counterproductive.

A complex application system, its performance often depends on the weakest application system of the internal resources. This contains many factors other than hardware, such as database design aspects including table design, index design, table association structure design, lock latch contention, etc., middleware deployment including message queue processing mechanism, connection pool maintenance, etc., as well as program development which involves more factors. These factors other than hardware are often the biggest cause of constricts for the system performance. Database application with a deadlock in the cloud computing end can only affect a lot of end users.

As a third party testing institutions which the authors worked for in China, many application performance problems often come from the internal system, such as a bad design makes a huge system paralysis. In this case, it is effort wasted to deploy more hardware. Even for hardware bottlenecks, cloud computing service mode may not give a satisfactory solution. In a large enterprise application, for example, concerning information security, its data center usually in the private network, and only the application access layer is deployed in the cloud serve to solve the problem of user access bandwidth. So disk IO performance bottlenecks of the intranet data center tends to restrict the overall performance improvement and this case is not rare. 


\section{B. Poor Development Of Cloud Services Platform Can Cause Huge Waste Of Public Resources}

More than 20 cities in China now make the cloud computing industry development as the key industry, but some of them just follow the policy. Although the policy guidance will promote cloud computing to be the primary manner of information processing in the future, the enterprise should achieve profit growth, and can further develop new technologies in the case of certain conditions satisfied. A lot of repeated investment will only cause the waste of public resources.

\section{1) Cloud computing consumes huge energy}

On May 26, in the "clean energy BBS of 2012", Zhang Guobao, the Minister of China's national energy bureau said that the data center as the physical foundation of "cloud computing" is very energy.

Zhang stressed that with his personal knowledge of China Unicom data center power consumption figures [2], the center consume 9.9 billion kilowatt hours of electricity each year. According the effectiveness of standard coal in China, it needs to consume 920000 tons of standard coal to provide enough power for China Unicom data center power. Corresponding to China Unicom, China Telecom data center power consumed 11.2 billion $\mathrm{KWH}$, consuming 1.0295 million tons of standard coal in total.

According to a Greenpeace report, in 2020, the world's major IT operators "cloud computing", including data processing and telecommunication network, will need nearly 2 trillion kilowatt-hours of energy consumption, more than the combined energy consumption of the 4 countries which are Germany, France, Canada and Brazil. In 2011, the China data center consumes a total power of 70 billion $\mathrm{KWH}$, accounting for $1.5 \%$ of the whole nation power consumption, equivalent to the total electricity consumption of Tianjin in 2011.

It is reported by the New York Times, "energy efficiency is actually very low in providing basic support for the cloud service data center ". According to the relevant test results, the data center use only about $10 \%$ of electricity to process its data, while the remaining $90 \%$ power is used to maintain the server open circuit and other auxiliary equipment. In order to prove that data center is a power-hungry, the report also cites both Google and Facebook as an example. The global data centers of Google consume about 300 million degrees of electricity every year, which is more than the annual electricity consumption of thirty thousand households in American. While Facebook is slightly better, consumes around 60 million degrees of electricity in one year.

\section{2) Huge consumption of network resources}

The application of Internet of things technology is developed step by step. The 12th five-year plan of Internet of things in China is to support application demonstration project for key fields, including intelligent industry, intelligent agriculture, intelligent logistics, intelligent transportation, smart grid, smart environmental protection, intelligent security, intelligent medical and intelligent home. Perception layer of the Internet of things needs a lot of data exchanged by the network layer and application layer, so basic transmission network demand is huge. Cloud computing concept is still in its early stage, along with policy support and industrial development, the cloud computing market will exceed 100 billion Yuan in China in 2013.

Cloud computing, especially cloud storage, needs huge bandwidth. At the same time, for a long time, cloud computing, big data with emerging technologies such as data center in the corresponding data processing, storage, and software system, gradually enrich the application of traditional data centers, and these applications put forward higher requirements for the network. Huge consuming of network bandwidth is a drain on public network resources.

According to a test project in my center recently, a booking business system with 300 servers, under the condition of 200000 people's visiting, costs up to 80 GBPS of network bandwidth. With tens of millions of people's visiting, such as taobao and amazon system, the occupations of public network bandwidth resources are more obvious, especially when the international export bandwidth is only TB level in China at present, the public network resources is particularly valuable.

For a society, we should not only consider whether the cloud computing platform is efficiency, but also the energy question, public social costs, such as the network bandwidth resource contention issues. And it is no meaning for the macro social if the efficiency increasing while the social costs also increasing.

3) The cases of redundant construction

A problem worthy of attention is that the countermeasures for foreign cloud computing service providers entering the Chinese domestic market in the cloud computing industry development process.

Admittedly, cloud computing abroad is earlier and superior than that in China. Now our technology users and promoters have contradiction. Promoters aware of "cloud" earlier than the users and have business interests, so when considering local interests, they don not consider to decrease the total social cost sometimes. "Cloud computing" aboard come in to China by the way of "in its original form", and the this will always help the foreign companies succeed in business, while we get just a shell that means the hardware only, not soft power such as virtualization technology. And all levels of the government's excessive zeal in China may not only enlarge the inherent data security issues, but also cause huge waste of resources and public resources diverted.

\section{HeAlthy Services In The Cloud MeAns GoOd APPLICATION ECOSYSTEM}

The cloud is not the server, but the service. Cloud computing market potential is tremendous in China, but we should pay our attention to some problems. Such as the cloud computing industry focus on the data center construction at present in China. Infrastructure is important, but cloud computing services are the application eventually. After integration, the upper of cloud computing technology focuses on the cloud applications software. If there is no proper service and profit model, cloud computing center could even become a decoration consuming a lot of electric power. The persons inside this industry said, a many of cloud computing center in China is low efficiency in resources usage, some even have no virtualization, which is completely contrary to high efficiency, energy saving, low cost of cloud computing. The operating income of some computing center currently 
even cannot afford their electricity fee. Without the application, the Data Center will be the only a center with no data.

If only considering the concept of cloud computing industry without the actual development of it, and inadequately considering each link of the industrial chain, under the idea of software important, the introduction of cloud computing services are likely to become a display and promotion center for the foreign equipment and product, and is kidnapped by the foreign manufacturer's technology. In this way, it not only can enlarge security and privacy problems born with cloud computing, but also will cause a huge waste of public resources in China and even may suppress the domestic independent innovation opportunities.

Based on the integration of existing resources, finding out a good business model and building a good quality of cloud applications is the way to make the convenient and efficient of the cloud computing into the end user's experiences.

\section{REFERENCES}

[1] The people's Republic of China National Economic and social development of the Twelfth Five Year Plan Outline [OL]. http://www.ce.cn/macro/more/201103/16/t20110316_22304698.shtml

[2] The 2012 U.S. - China Clean Energy Forum [OL]. http://www.compuware.com/application-performance-management 